\title{
THE APPLICATION OF THE BOUNDARY ELEMENT METHOD TO SOLVE VISCOUS FLOW PROBLEMS BASED ON PRIMITIVE VARIABLES FORMULATION
}

\author{
M. F. C. L. Santos \\ UNESP - Faculdade de Engenharia de \\ Guaratinguetá \\ Av. Dr. Ariberto Pereira da Cunha, 333 \\ 12.516-410 - Guaratinguetá - SP - Brazil \\ flacaz@feg.unesp.br \\ J. R. Barbosa \\ H. F. F. M Carneiro \\ Instituto Tecnológico de Aeronáutica

\begin{abstract}
The boundary element method is applied to the solution of incompressible fluid flow problems governed by the continuity and Navier-Stokes equations. The differential equations are transformed into integral equations. Indication of the transformation is given in detail. Application to simple flow cases such as the driven cavity and forward facing step is presented. Convergence difficulties are indicated, which have limited the applications
\end{abstract} \\ to flows of low Reynolds numbers.
} CTA-ITA-IEM/IEME 12.228-900 - São José dos Campos - SP Brazil

barbosa@mec.ita.br
Key Words: Boundary element, Navier-Stokes, numerical methods

\section{INTRODUCTION}

The Boundary Element Method (BEM) is a relatively recent technique that has found great application in several fields of engineering. This technique has received increasing attention of a great number of researchers in mechanics of fluids field, as one can see by the increasing number of articles published in the literature. Certainly, that is the area where BEM users are facing one of the biggest obstacles, related to the strong non-linearity of the convective terms, that has been making the convergence of the solutions for high Reynolds number flows a difficult task. Besides, many other aspects related to the numerical implementation have also contributed to limit the convergence of results and, in some cases, to reduce accuracy, such as, hyper and super singular integrals, quasi-singular integrals, corner effects, discretization effects and so on.

This work aims at the application of this method to the solution of steady state, two-dimensional laminar flow of incompressible viscous fluid. Option was made to formulate the problem in primitive variables (pressure and velocity) that, although it seems to be more stable, it is recognized as one of the most difficult approaches since it is more involving from the mathematical point of view, in order to obtain the integral equations. The integral equations were derived from the Navier-Stokes equations, using the Weighting Residual Method. All variables involved in the solution were obtained through the integral equations, except for the velocity component derivatives in $\mathrm{x}$ - and $\mathrm{y}$-directions that were obtained by the Finite Difference Method for the sake of simplicity. The boundary was discretized by constant type elements, and linear triangular cells were adopted in the domain discretization.

The need for solution of the system of partial differential equations which model the flow of a fluid in channels like pipes, blade passages, nozzles and others, appeared with the very day the fluid flow was modeled.
The difficulties involved in obtaining closed solutions, even for very simple flows, required the development of clever techniques but only with the application of numerical solutions to that system of equations, some flows of practical interest were calculated.

Several computational techniques have been used: finite difference, finite element, finite volume and boundary element to name the most known. As new algorithms were discovered and faster computer was produced, each of those methods evolved in all areas in the past years. Finite difference methods have been, and already are, implemented to solve flow problems. Finite elements gained attention in the past decades; in the seventies it was still crawling. Both are bases for commercial codes for the solution of flows of almost every kind. Computer effort has been limiting the application of the numerical methods in the sense that every new method of solution discovered claims reduction in CPU time and storage requirements. But the reasons that these methods are so CPU time and storage hunger are intrinsic.

The boundary element method, nevertheless, has progressed differently depending on the areas where it has been applied, fastest in areas related to solid mechanics, slowest in the ones related to fluid mechanics.

The method is similar to the finite element method. While the latter solves an algebraic system of equations obtained from integrals over elements in which the volume is divided (finite element), the former solves integrals over the boundary of such elements (boundary element). Surface integrals are obtained by transformation of volume integrals applying Green-Gauss theorem. So, the boundary element technique reduces of one the number of dimensions of the problem.

Despite integral methods were available many decades ago for the application to flow problems of practical interest, a comprehensive study of the formulation and application to flow problems are still being considered 
more recently, as they are expected to alleviate the storage and hopefully CPU time sensibly, because roughly the degrees of freedom is reduced in the boundary element method.

Although this apparent advantage, requiring less computational effort when volume integrals are transformed into surface integrals, some disadvantages arise, such as higher mathematical complexity in order to get an usable computational formulation; the need for the calculation of infinite integrals; fully populated matrices whose inversion is not so simple as the band matrices in the finite difference and finite element schemes.

In order to validate the computational model the driven cavity and step channel flow problems were adopted as test cases. Most importantly, an attempt to extend the application of the method to solve the cascade flow of axial turbomachinery was made, which represented the most challenging target of this work. The main difficulty faced was due to the lack of convergence of results for high Reynolds number flows.

\section{STATEMENT OF THE PROBLEM}

Let $\Omega$ and $\Gamma$ be the domain and respective boundary. The domain can be a compressor inter-blade passage and the boundary the solid surfaces of two consecutive blades in a cascade and the hub and casing surfaces, for the example of one of the geometry of interest. This is considered a very complex flow which even the most developed numerical methods have problems in predicting accurate solution. The fluid, in this case, could be air, considered as a perfect gas, compressible and viscous.

Let $(\mathrm{x}, \mathrm{y})$ be a point of $\Omega$. in $\mathrm{R}^{2}$. An incompressible steady flow can be modeled by the conservation equations of mass, $\mathrm{x}$ - and y-momentum, as given by Eqs. 1, 2 and 3, respectively, according to Santos ${ }^{1}$ and Barbosa and Santos ${ }^{2}$ :

$$
\frac{\partial \mathrm{u}}{\partial \mathrm{x}}+\frac{\partial \mathrm{v}}{\partial \mathrm{y}}=0
$$

$\mu \nabla^{2} u+\mu \frac{\partial}{\partial x}\left(\frac{\partial u}{\partial x}+\frac{\partial v}{\partial y}\right)-\frac{\partial P}{\partial x}=\rho\left(u \frac{\partial u}{\partial x}+v \frac{\partial u}{\partial y}\right)$

$\mu \nabla^{2} v+\mu \frac{\partial}{\partial y}\left(\frac{\partial u}{\partial x}+\frac{\partial v}{\partial y}\right)-\frac{\partial P}{\partial y}=\rho\left(u \frac{\partial v}{\partial x}+v \frac{\partial v}{\partial y}\right)$

The variables are $\mathrm{u}, \mathrm{v}$

and y directions;

$$
\begin{aligned}
& \rho, \mathrm{P} \\
& \mu, v
\end{aligned}
$$

viscosities;

$\mathrm{k}, \mathrm{c}_{\mathrm{v},} \mathrm{c}_{\mathrm{p}} \quad$ thermal conductivity and specific heats at constant volume and pressure.

In order to generalize the problem, let the following change of variables (Eq. 4) take effect in the conservation equations:

$$
\begin{gathered}
\bar{x}=\frac{x}{L} \quad \overline{\mathrm{y}}=\frac{\mathrm{y}}{\mathrm{L}} \quad \overline{\mathrm{u}}=\frac{\mathrm{u}}{\mathrm{V}_{\infty}} \\
\overline{\mathrm{v}}=\frac{\mathrm{v}}{\mathrm{V}_{\infty}} \\
\overline{\mathrm{P}}=\frac{\mathrm{P}-\mathrm{P}_{\infty}}{\rho \mathrm{V}_{\infty}^{2}} \quad \bar{\rho}=\frac{\rho}{\rho_{\infty}} \\
\bar{\mu}=\frac{\mu}{\mu_{\infty}} \quad \bar{v}=\frac{v}{v_{\infty}} \quad \operatorname{Re}=\frac{\rho_{\infty} \mathrm{V}_{\infty} \mathrm{L}}{\mu_{\infty}} \\
\mathrm{V}_{\infty}=\sqrt{\mathrm{u}_{\infty}^{2}+\mathrm{v}_{\infty}^{2}}
\end{gathered}
$$

where the properties with the infinity symbol index refer to the far stream condition. Re and Pr are the Reynolds and Prandtl numbers, respectively.

The equations of conservation of mass, $\mathrm{x}-$ and $\mathrm{y}$ momentum become, after the change of variables, appropriate collection of terms and dropping the overbar:

$\frac{\partial \mathrm{u}}{\partial \mathrm{x}}+\frac{\partial \mathrm{v}}{\partial \mathrm{y}}=0$

$\nabla^{2} u+\frac{\partial}{\partial x}\left(\frac{\partial u}{\partial x}+\frac{\partial v}{\partial y}\right)-\operatorname{Re} \frac{\partial P}{\partial x}=\operatorname{Re}\left(u \frac{\partial u}{\partial x}+v \frac{\partial u}{\partial y}\right)$

$\nabla^{2} v+\frac{\partial}{\partial y}\left(\frac{\partial u}{\partial x}+\frac{\partial v}{\partial y}\right)-\operatorname{Re} \frac{\partial P}{\partial y}=\operatorname{Re}\left(u \frac{\partial v}{\partial x}+v \frac{\partial v}{\partial y}\right)$

The variables of the problem, considering a perfect gas fluid, are $\mathrm{u}, \mathrm{v}$ and $\mathrm{P}$. Therefore, the system of partial differential equations can be rewritten in matrix form as Eq. 8:

$\mathrm{L}_{\mathrm{IJ}} \mathrm{U}_{\mathrm{J}}=\mathrm{B}_{\mathrm{I}}$

where $\mathrm{L}$ is a linear partial differential operator, $\mathrm{U}$ is the unknown vector $\left[\begin{array}{lll}\mathrm{u} & \mathrm{V} & \mathrm{P}\end{array}\right]^{\mathrm{t}}$ and $\mathrm{B}$ the vector of nonlinear terms. Depending on the assumptions made, $\mathrm{L}$ and B can take different forms. For instance, vector B can be linearised and its linear terms included in L. I and $\mathrm{J}$ denotes line and columns, respectively, of the matrices.

Let, for the moment, all non-linear terms be included into B. Then

$$
\mathrm{U}_{\mathrm{J}}=\left[\begin{array}{lll}
\mathrm{u} & \mathrm{v} & \mathrm{P}
\end{array}\right]^{\mathrm{t}}
$$

For the sake of simplification, let the differential operators, defined by Eq. 10, be incorporated in the equations, 


$$
\begin{array}{r}
\mathrm{D}_{1}=\frac{\partial}{\partial \mathrm{x}} \quad \mathrm{D}_{2}=\frac{\partial}{\partial \mathrm{y}} \\
\nabla^{2}=\frac{\partial^{2}}{\partial \mathrm{x}^{2}}+\frac{\partial^{2}}{\partial \mathrm{y}^{2}}
\end{array}
$$

and ignore the terms containing the variation of $\mu$ and $\mathrm{k}$.

Then,

$\mathrm{L}_{\mathrm{IJ}}=\left[\begin{array}{ccc}\nabla^{2}+\mathrm{D}_{1} \mathrm{D}_{1} & \mathrm{D}_{1} \mathrm{D}_{2} & -\operatorname{Re} \mathrm{D}_{1} \\ \mathrm{D}_{2} \mathrm{D}_{1} & \nabla^{2}+\mathrm{D}_{2} \mathrm{D}_{2} & -\operatorname{Re} \mathrm{D}_{2} \\ \mathrm{D}_{1} & \mathrm{D}_{2} & 0\end{array}\right]$,

$\mathrm{U}_{\mathrm{J}}=\left[\begin{array}{c}\mathrm{u} \\ \mathrm{v} \\ \mathrm{P}\end{array}\right] \quad$ and
$\mathrm{B}_{\mathrm{I}}=\left[\begin{array}{c}\operatorname{Re}\left(\mathrm{u} \frac{\partial \mathrm{u}}{\partial \mathrm{x}}+\mathrm{v} \frac{\partial \mathrm{u}}{\partial \mathrm{y}}\right) \\ \operatorname{Re}\left(\mathrm{u} \frac{\partial \mathrm{v}}{\partial \mathrm{x}}+\mathrm{v} \frac{\partial \mathrm{v}}{\partial \mathrm{y}}\right) \\ 0\end{array}\right]$

One looks for the solution of Eq. 8 subject to the boundary conditions appropriate for the problem. Those conditions will be stated later, when the applications are shown.

\section{THE METHOD}

There is no known analytical solution for the Eq. 8 . Let $\widetilde{U}$ be an approximate solution in the sense

$\mathrm{L} \widetilde{U}-\mathrm{B}=\mathrm{R} \cong 0$

that is, $\widetilde{U}$ differs very little from $U$ but it is not equal to $U$. One seeks to find $\widetilde{U}$ such that

$$
\iint_{\Omega}(\mathrm{LU}-\mathrm{B}) \mathrm{Wd} \Omega=\iint_{\Omega} \mathrm{RW} \mathrm{d} \Omega=0
$$

where $\mathrm{W}$ is a weight function.

The name "boundary element method" comes after the way $\mathrm{W}$ is defined and the way Eq. 13 is solved.

\section{THE WEIGHT FUNCTION}

The weight function, usually called fundamental solution, is determined from Eq. 14. Let

$$
\mathrm{AX}=\mathrm{E} \Psi
$$

be a linear system of equations where $\mathrm{A}$ and $\mathrm{E}$ are known matrices, $\Psi$ is a multiplying scalar and $\mathrm{X}$ the unknown solution vector. Vector $\mathrm{X}$ is obtained multiplying both members of Eq. 14, to the left, by $\mathrm{A}^{-1}$, where $\mathrm{A}^{-1}=[\operatorname{det} \mathrm{A}]^{-1}[\operatorname{cof} \mathrm{A}]^{\mathrm{t}}$.

Let $\mathrm{W}$ be calculated after the auxiliary problem given by Eq. 17, deduced from Eqs. 15 to 17 below.

$$
\mathrm{L}^{*} \mathrm{~W}=\delta(\mathrm{X}-\mathrm{Y})
$$

Then

$$
\mathrm{W}=\left(\mathrm{L}^{*}\right)^{-1} \delta(\mathrm{X}-\mathrm{Y})=\left(\operatorname{cofL}^{*}\right)^{\mathrm{t}}\left(\operatorname{det} \mathrm{L}^{*}\right)^{-1} \delta(\mathrm{X}-\mathrm{Y})
$$

or

$$
\mathrm{W}=\left(\operatorname{cofL}^{*}\right)^{\mathrm{t}} \Phi^{*}, \quad \text { where } \Phi^{*}=\left(\operatorname{det} \mathrm{L}^{*}\right)^{-1} \delta(\mathrm{X}-\mathrm{Y})
$$

Finally,

$$
\left(\operatorname{det} L^{*}\right) \Phi^{*}=\delta(\mathrm{X}-\mathrm{Y})
$$

Thus, if one finds the function $\Phi^{*}$. defined by the characteristic Eq. 17, one builds the weight function. These processes are neither straightforward nor easy.

Matrices $\mathrm{L}^{*}=\overline{\mathrm{L}}_{\mathrm{IJ}}$ and $\mathrm{M}_{\mathrm{JK}}^{*}=\left(\operatorname{cof} \overline{\mathrm{L}}_{\mathrm{IJ}}\right)^{\mathrm{t}}$, the adjoint of $\mathrm{L}_{\mathrm{IJ}}$ and its transposed cofactor are:

$$
\begin{aligned}
\overline{\mathrm{L}}_{\mathrm{IJ}} & =\left[\begin{array}{ccc}
\nabla^{2}+\mathrm{D}_{1} \mathrm{D}_{1} & \mathrm{D}_{2} \mathrm{D}_{1} & -\mathrm{D}_{1} \\
\mathrm{D}_{1} \mathrm{D}_{2} & \nabla^{2}+\mathrm{D}_{2} \mathrm{D}_{2} & -\mathrm{D}_{2} \\
\operatorname{Re} \mathrm{D}_{1} & \operatorname{Re} \mathrm{D}_{2} & 0
\end{array}\right] \\
\mathrm{M}_{\mathrm{JK}}^{*} & =\left[\begin{array}{ccc}
\operatorname{Re} \mathrm{D}_{2} \mathrm{D}_{2} & -\operatorname{Re} \mathrm{D}_{2} \mathrm{D}_{1} & \mathrm{D}_{1} \nabla^{2} \\
-\operatorname{Re} \mathrm{D}_{1} \mathrm{D}_{2} & \operatorname{Re} \mathrm{D}_{1} \mathrm{D}_{1} & \mathrm{D}_{2} \nabla^{2} \\
-\operatorname{Re} \mathrm{D}_{1} \nabla^{2} & -\operatorname{Re} \mathrm{D}_{2} \nabla^{2} & 2 \nabla^{2} \nabla^{2}
\end{array}\right]
\end{aligned}
$$

$$
\begin{aligned}
& \text { so that } \\
& \operatorname{det}\left[\overline{\mathrm{L}}_{\mathrm{IJ}}\right]=\operatorname{Re} \nabla^{2}\left(\nabla^{2}\right)=\operatorname{Re} \nabla^{4}
\end{aligned}
$$

The Eq. 18 has the solution given by Eq. 21:

$$
\Phi^{*}(X, Y)=\frac{1}{\operatorname{Re}}\left(\frac{1}{8 \pi} r^{2} \ln r\right)
$$

Therefore, the weight function is determined. Next step is the integration of Eq. 13. 


\section{DISCRETIZATION}

The Green-Gauss Theorem applied to Eq. 13 allows the domain integral be transformed into an integral over the boundary $\Gamma$. This contour $\Gamma$ is usually divided into $n_{e}$ boundary elements. Therefore

$$
\iint_{\Omega}(\mathrm{LU}-\mathrm{B}) \mathrm{Wd} \Omega=\sum_{\mathrm{e}=1 \Gamma_{\mathrm{e}}}^{\mathrm{n}_{\mathrm{e}}} \iint_{\mathrm{e}} \mathrm{F}(\mathrm{U}, \mathrm{B}, \mathrm{W})_{\mathrm{e}} \mathrm{d} \Gamma_{\mathrm{e}}=0
$$

which indicates that the system of differential equations (Eq. 12) has been transformed into a system of algebraic equations (Eq. 22) that involves the values of the variables at each boundary element. The solution on the boundary may be found provided the values of the variables at the elements on the boundary are determined.

After the application of the Green-Gauss theorem and integration by parts over $\Omega$, Eq. 23 , that holds also for every boundary element, is determined:

$$
\begin{aligned}
& \iint_{\Omega} \mathrm{U}_{\mathrm{IJ}}\left(\overline{\mathrm{L}}_{\mathrm{IJ}} \mathrm{W}_{\mathrm{IK}}^{*}\right) \mathrm{d} \Omega= \\
& \int_{\Gamma}\left\{\mathrm{u}_{\mathrm{i}}(\mathrm{X}) \mathrm{S}_{\mathrm{iK}}^{*}(\mathrm{X}, \mathrm{Y})-\tau_{\mathrm{i}}(\mathrm{X}) \mathrm{W}_{\mathrm{Ik}}(\mathrm{X}, \mathrm{Y})\right\} \mathrm{d} \Gamma(\mathrm{X}) \\
& +\iint_{\Omega} \mathrm{B}_{\mathrm{I}}(\mathrm{X}) \mathrm{W}_{\mathrm{Ik}}(\mathrm{X}, \mathrm{Y}) \mathrm{d} \Omega(\mathrm{X})
\end{aligned}
$$

In a limit process at which $\mathrm{Y}$ approaches $\mathrm{X}$, considering the singularities of $\mathrm{W}_{\mathrm{iK}}^{*}$, one arrives at Eq. 24, usually called the boundary integral equation:

$$
\begin{aligned}
& \mathrm{C}_{\mathrm{KI}}(\mathrm{Y}) \mathrm{U}(\mathrm{Y})=\iint_{\Gamma}\left\{\mathrm{u}_{\mathrm{i}}(\mathrm{X}) \mathrm{S}_{\mathrm{ik}}^{*}(\mathrm{X}, \mathrm{Y})-\tau_{\mathrm{i}}(\mathrm{X}) \mathrm{W}_{\mathrm{ik}}(\mathrm{X}, \mathrm{Y})\right\} \mathrm{d} \\
& +\iint_{\Omega} \mathrm{B}_{\mathrm{I}}(\mathrm{X}) \mathrm{W}_{\mathrm{Ik}}(\mathrm{X}, \mathrm{Y}) \mathrm{d} \Omega(\mathrm{X})
\end{aligned}
$$

where summation is implied by repeating index.

It is worth mentioning that the second member of Eq. 24 comprises integrals over the boundary and over the domain, these due to the non-linear convective terms.

$\mathrm{C}_{\mathrm{KI}}(\mathrm{y})$ is the tensor coefficient dependent on the boundary geometry. If $y$ lies over the boundary and the boundary is there regular, its value is $1 / 2$; if the point y lies in the domain, its value is 1 ; if $y$ is in the boundary and there is discontinuity of the derivative of $\Gamma$, then its value depends on the angle formed by the left and right tangents. Details of the terms of Eq. 23 are:

$$
\begin{aligned}
& s_{i K}^{*}(x, Y)=\left(-W_{3 K}^{*} \delta_{i j}+R e \widetilde{u}_{j} W_{i K}^{*}+w_{i K, j}^{*}+w_{j K, i}^{*}\right) h_{j} \\
& \text { and } \\
& \tau_{i}(x)=\left(-R e p \delta_{i j}+u_{i j}+u_{j i}\right) h_{j}
\end{aligned}
$$

where coma (,) indicates derivative with respect to the following index and summation is implied by repeating indices.

Selecting the constant element, one has

$$
\left\{\begin{array}{l}
u_{i}\left(\Gamma_{e}\right)=u_{i_{e}} \\
\tau_{i}\left(\Gamma_{e}\right)=\tau_{i_{e}}
\end{array}\right.
$$

Therefore, equation (24) becomes, after integration

$$
\begin{aligned}
& \mathrm{C}_{\mathrm{KI}}(\mathrm{Y}) \mathrm{U}_{\mathrm{I}}(\mathrm{Y})= \\
& \sum_{\mathrm{e}=1}^{\mathrm{n}_{2}} \int_{\Gamma}\left\{\mathrm{S}_{\mathrm{ik}}^{*}(\mathrm{X}, \mathrm{Y}) \mathrm{u}_{\mathrm{i}_{\mathrm{e}}}-\mathrm{W}_{\mathrm{ik}}(\mathrm{X}, \mathrm{Y}) \tau_{\mathrm{i}}\right\} \mathrm{d} \Gamma_{\mathrm{e}}+ \\
& +\iint_{\Omega} \mathrm{B}_{\mathrm{I}}(\mathrm{X}) \mathrm{W}_{\mathrm{Ik}}(\mathrm{X}, \mathrm{Y}) \mathrm{d} \Omega(\mathrm{X})
\end{aligned}
$$

It is worth noting that the terms corresponding to the variables values in each element can be factored so that the system of algebraic equations becomes then evident.

\section{IMPLEMENTATION}

Boundary

Let the boundary $\Gamma$ be divided into $\mathbf{m}$ constant elements, with the collocation points (nodes) placed at the mid point of each element. Application of Eq. 26 to those $\mathbf{m}$ nodes gives a set of $3 \mathbf{m}$ equations with $3 \mathbf{m}$ unknowns. For the solution of this system of equations two auxiliary matrices are assembled, for each element:

$\mathrm{G}_{\beta \alpha}(\mathrm{X}-\mathrm{Y})=\mathrm{S}_{\mathrm{ik}}^{*}(\mathrm{X}, \mathrm{Y})$

$\mathrm{H}_{\beta \alpha}(\mathrm{X}-\mathrm{Y})=\mathrm{W}_{\mathrm{ik}}(\mathrm{X}, \mathrm{Y})$

with $\alpha, \beta=1,2$.

from which is derived Eqs. 28.

$$
\begin{aligned}
& \mathrm{g}_{\alpha \beta}^{\mathrm{e}}(\mathrm{Y})=\int_{\Gamma_{\mathrm{e}}} \mathrm{G}_{\beta \alpha}(\mathrm{X}-\mathrm{Y}) \mathrm{d} \Gamma_{\mathrm{e}} \\
& \mathrm{h}_{\alpha \beta}^{\mathrm{e}}(\mathrm{Y})=\int_{\Gamma_{\mathrm{e}}} \mathrm{H}_{\beta \alpha}(\mathrm{X}-\mathrm{Y}) \mathrm{d} \Gamma_{\mathrm{e}}
\end{aligned}
$$

with $\alpha, \beta=1,2,3$.

Integrals of Eq. 28 are carried out numerically, using the Gauss method straight, when $X \neq Y$. When $X=Y$ or if $\mathrm{X} \approx \mathrm{Y}$ the integrands become infinite, requiring the calculation in the sense of Cauchy principal value. Several techniques are available to perform these calculations, all requiring careful calculation of such integrals.

\section{Domain}

To calculate the integrals over $\Omega$, the domain is divided into $\mathbf{M}$ elements by an appropriate net. Using the linearity of integrals, one obtains Eq. 28:

$$
\begin{aligned}
& \iint_{\Omega} \mathrm{B}_{\mathrm{I}}(\mathrm{X}) \mathrm{W}_{\mathrm{Ik}}(\mathrm{X}, \mathrm{Y}) \mathrm{d} \Omega(\mathrm{X})= \\
& \sum_{\mathrm{e}=1}^{\mathrm{M}}\left[\sum_{\mathrm{j}=1}^{\mathrm{J}} \omega_{\mathrm{j}}\left(\mathrm{B}_{\mathrm{I}} \mathrm{W}_{\mathrm{Ik}}(\mathrm{X}, \mathrm{Y})\right)_{\mathrm{j}}\right] \mathrm{S}_{\Omega_{\mathrm{e}}}
\end{aligned}
$$

where $\omega_{\mathrm{j}}$ is the Gauss weight function at point $\mathrm{j}, \mathrm{S}_{\Omega_{\mathrm{e}}}$ is 
the element area, and $\mathrm{J}$ is the number of Gauss integration points.

\section{Computer Program}

In order to implement a computer program, the following steps are suggested:

- discretization of the boundary in $\mathrm{n}_{\mathrm{e}}$ elements

- calculation of the geometric properties for the elements in the boundary

- assembly of matrices $\mathrm{g}_{\alpha \beta}^{\mathrm{e}}$ and $\mathrm{h}_{\alpha \beta}^{\mathrm{e}}$ for each element and

- assembly of matrices $\mathrm{G}$ and $\mathrm{H}$ for all elements in the contour.

To do this, define the vectors of unknown variables at the boundary:

$$
\begin{aligned}
& \delta=\left[\begin{array}{lllll}
\mathrm{u}_{1} & \mathrm{v}_{1} & \cdots & \mathrm{u}_{\mathrm{m}} & \mathrm{v}_{\mathrm{m}}
\end{array}\right]^{\mathrm{t}} \\
& \tau=\left[\begin{array}{lllll}
\tau_{11} & \tau_{21} & \cdots & \tau_{1 \mathrm{~m}} & \tau_{2 \mathrm{~m}}
\end{array}\right]^{\mathrm{t}}
\end{aligned}
$$

and write Eq. 29 in the form

$$
\mathrm{C} \delta+\mathrm{H} \delta=\mathrm{G} \tau+\mathrm{D}
$$

or, making $\overline{\mathrm{H}}=\mathrm{C}-\mathrm{H}$, one arrives at Eq. 32 .

$$
\overline{\mathrm{H}} \delta=\mathrm{G} \tau+\mathrm{D}
$$

- application of the boundary conditions to Eq. 32.

To do this, note that elements of $\delta$ and $\tau$ have some values prescribed. Rearrange $\delta$ and $\tau$ such that the unknown variables come first and then the prescribe values, as indicated by Eq. 33 .

$$
\begin{aligned}
\delta=\left[\begin{array}{ll}
\delta_{u} & \delta_{p}
\end{array}\right]^{t} & \\
& \tau=\left[\begin{array}{ll}
\tau_{u} & \tau_{p}
\end{array}\right]^{t} .
\end{aligned}
$$

- rearrangement of matrices $\overline{\mathrm{H}}, \mathrm{G}$ and $\mathrm{D}$ accordingly, such that Eq. 33 arrives:

$\left[\begin{array}{ll}\overline{\mathrm{H}}_{\mathrm{uu}} & \overline{\mathrm{H}}_{\mathrm{up}} \\ \overline{\mathrm{H}}_{\mathrm{pu}} & \overline{\mathrm{H}}_{\mathrm{pp}}\end{array}\right]\left[\begin{array}{l}\delta_{\mathrm{u}} \\ \delta_{\mathrm{p}}\end{array}\right]=\left[\begin{array}{ll}\overline{\mathrm{G}}_{\mathrm{uu}} & \overline{\mathrm{G}}_{\mathrm{up}} \\ \overline{\mathrm{G}}_{\mathrm{pu}} & \overline{\mathrm{G}}_{\mathrm{pp}}\end{array}\right]\left[\begin{array}{l}\tau_{\mathrm{u}} \\ \tau_{\mathrm{p}}\end{array}\right]+\left[\begin{array}{c}\mathrm{D}_{\mathrm{u}} \\ \mathrm{D}_{\mathrm{p}}\end{array}\right]$

- rearrangement of Eq. 34 such that only the unknowns are in the left side, that is,

$$
\mathbf{A X}=\mathbf{B P}+\mathrm{D}
$$

Matrix A is almost fully populated (dense) so that inversion is time consuming. Its inversion may be carried out by the Gauss elimination algorithm. Solution of Eq. 35 gives the values of the unknowns at the boundary.
- internal nodes

Equation 30 are used for the calculation of the internal nodes, for which the constants in $\mathrm{C}$ are equal to 1 . The solution at the internal nodes starts with a guess of the flow field and imposition of the boundary conditions.

\section{Grid Generation}

For the problems indicated below, the grid was generated using orthogonal straight lines linking the opposite surfaces, which were divided into a number of equal elements. For complex boundaries it is needed a special grid generation routine. This subject is not discussed in this work, although it may be a very complex task.

\section{APPLICATION}

To demonstrate the applicability of the method, the flow facing a forward step and the driven cavity flow were selected.

\section{Flow in a box with moving slid}

The driven cavity, that is, the flow in a box with the upper surface moving to the right, was tested using the software developed. A picture of the flow in the box is shown in Fig. 1 below. The boundary conditions are the no-slip conditions in the box boundaries, that is, zero at the nonmoving surfaces and the velocity of the moving slid at the upper surface. Tests were carried out with different grids to check the sensitivity of the algorithm to the grid size. Comparison of the results were carried out with the published data of Tosaka et $\mathrm{al}^{3}$, as indicated in Fig. 2, for the position $\mathrm{x}=0.5$, Reynolds number of 100 .

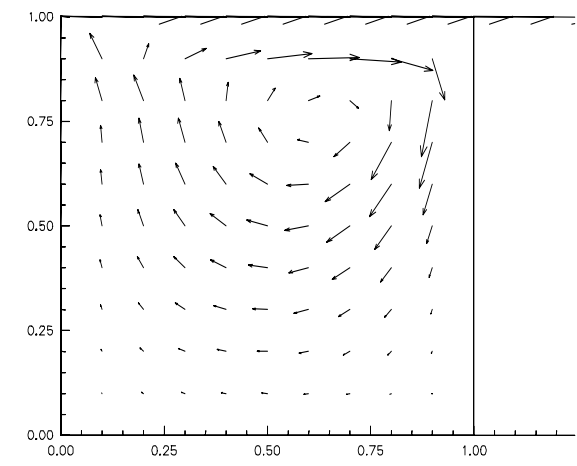

Figure 1. Driven cavity flow - Velocity vector field. 


\section{CIÊNCIA/SCIENCE}

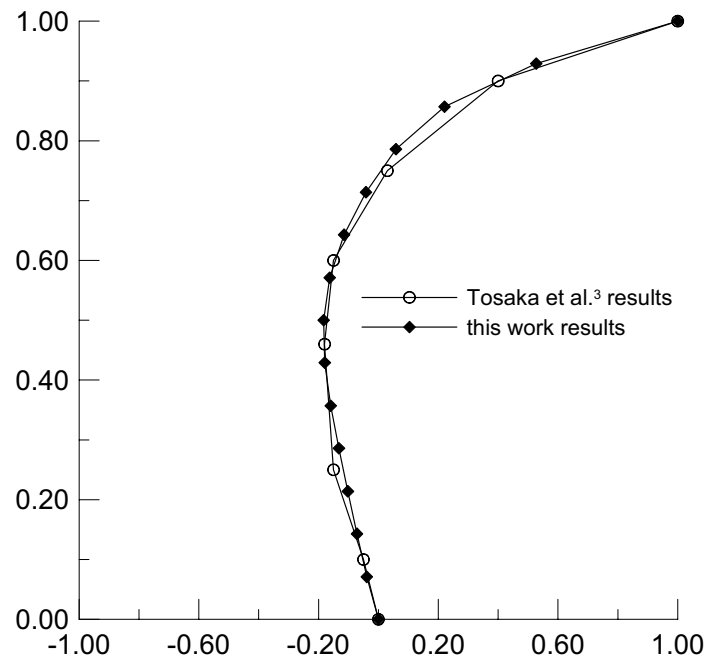

Figure 2. Velocity profile at the vertical center line of the cavity. $(\operatorname{Re}=100)$

\section{Flow in a stepped channel}

The flow in a forward facing step is shown in Figure 3 below, for the boundary conditions of no-slip on solid surfaces and parabolic profile for the axial velocity at channel inlet. The results shown refer to a grid of $17 \times 25$ points, Reynolds number of 20.

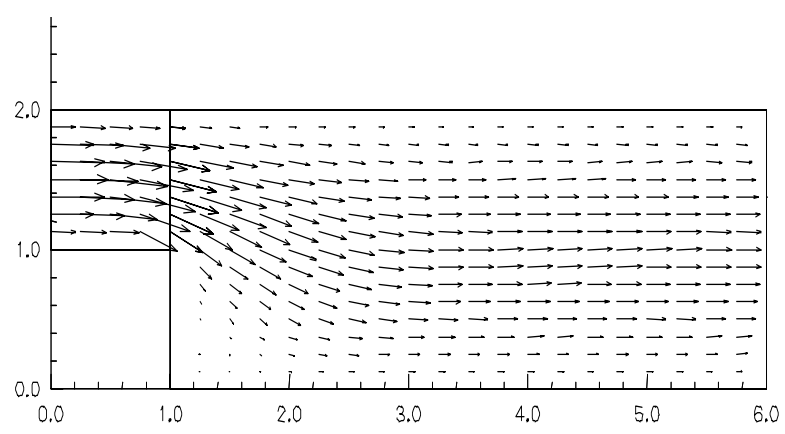

Figure 3. Channel flow - Velocity vector field

\section{REFERENCES}

Barbosa, J. R., Santos, M. F. C. L., 1998, "The Boundary Element Method in Fluid Mechanics", in: LATCYM 98, Vol. III, pp. 710-715.

Santos, M. F. C. L., 1998, Boundary Element Method Applied to Fluid Flow Problems, Ph.D. Thesis (in Portuguese), Instituto Tecnológico de Aeronáutica, São Paulo, Brazil.

Tosaka, N., Kakuda, K., Onishi, K., 1985, "Boundary Element Analysis of Steady Viscous Flows Based on P-VU Formulation", in: Proc. 7nth Conference of BEM in Engng., Lake Como, pp:71-80(9), Italy.

Tosaka, N., 1989, "Integral Equations Formulation with the Primitive Variables for Incompressible Viscous Fluid Flow Problems", Computational Mechanics, Vol. 4, pp:89-103.

\section{CONCLUSION}

The boundary element method can be applied to the calculation of compressible viscous flows as demonstrated through the above examples. Although a very coarse grid was used, the results are quite satisfactory, capturing regions of reverse flows as in the case of the stepped channel. CPU time was not recorded for this work but, comparing with other finite difference schemes used for the same purpose, it is considerably lower. It is therefore important to investigate other applications such as the flow in blade passages, the ultimate goal for the research under way. 